International Journal of Energy and Statistics Vol. 4, No. 4 (2016) 1699001 (3 pages)

(C) Institute for International Energy Studies DOI: $10.1142 / \mathrm{S} 2335680416990015$

\title{
Author index (Volume 4)
}

Abdollahzade, M., see Sharafi, A.

Alexopoulos, T. A. \& Thomakos, D. D., Functional smoothing for risk management of energy assets

Amalnick, M. S., see Sharafi, A.

Anbazhagan, S., Forecasting models for vertically bundled electricity market prices - A review and future trend

Arezzo, M. F., see Bramati, M. C.

Atchonouglo, K., see N'wuitcha, K.

Banna, M., see N'wuitcha, K.

Bramati, M. C., Arezzo, M. F. \& Pellegrini, G., Short-term wind power forecasting based on dynamic system of equations

Chavan, K. \& Gulhane, N., Performance investigation of compound parabolic collector system with triangular receiver with and without internal fins

Chen, W., see Li, W.

Cyrino Oliveira, F. L., see Maçaira, P. M.

Daud, H., see Khan, Z.

Dobrev, I., see

Noura, B.
2 (2016) 1650007

2 (2016) 1650006

2 (2016) 1650007

3 (2016) 1650011

3 (2016) 1650012

1 (2016) 1650003

1 (2016) 1650003

3 (2016) 1650012

1 (2016) 1650004

3 (2016) 1650015

2 (2016) 1650008

4 (2016) 1650016

3 (2016) 1650013
Engström, J., see Li, W.

3 (2016) 1650015

Fallahigilan, S. \& Sayyareh, A., Finite mixture model selection for total energy consumption

Farrokhifar, M., see Latifi, $\mathrm{H}$.

2 (2016) 1650009

1 (2016) 1650002

Fianu, E. S., The delay vector variance method and the recurrence quantification analysis of energy markets

1 (2016) 1650001

Fotuhi-Firuzabad, M., see Khan, Z.

Ghodsi, M., see Huang, X.

4 (2016) 1650016

4 (2016) 1650017

Ghosh, S., Majumder, M. \& Pal, M., Development of performance index for evaluation of small scale hydro power plants by neural network and multi criteria decision making

Gulhane, N., see Chavan, K.

4 (2016) 1650019

1 (2016) 1650004

Hassani, H. \& Silva, E.

S., Forecasting energy data with a time lag into the future and Google trends

4 (2016) 1650020

Huang, X. \& Ghodsi, M., A novel mutual association measure based on eigenvalue-based criterion 
Igo, S. W., see N'wuitcha, K.

Iranmanesh, H., see Sharafi, A.

Isberg, J., see Li, W. Jenkins, D. P., see Patidar, S.

Khan, Z., Razali, R. B., Daud, H., Nor, N. M.,

Fotuhi-Firuzabad, M. \& Krebs, K. L., Bad data detection in power system state estimation based on generalized likelihood ratio test

Khelladi, S., see Noura, B.

Kisaka, M. O., see Mbaka, C. K.

Krebs, K. L., see Khan, Z.

Kunst, R., Moghaddam, H. \& Wirl, F., Drivers and obstacles to biofuel: A dynamic panel data approach to selected European union countries

Latifi, H., Farrokhifar, M., Safari, A. \& Pournasir, S., Optimal sizing of combined heat and power generation units using of MPSO in the Besat Industrial Zone

Leijon, M., see Li, W.

Li, W., Isberg, J., Chen, W., Engström, J., Waters, R., Svensson, O. \& Leijon, M., Bivariate joint distribution modeling of wave climate data using a copula method

Maçaira, P. M. \& Cyrino Oliveira, F. L., Another look at SSA.Boot forecast accuracy $\mathbf{1}(2016) 1650003$
$\mathbf{2}(2016) 1650007$
$\mathbf{3}(2016)$
$\mathbf{3}(2016) 1650015$

4 (2016) 1650016

3 (2016) 1650013

2 (2016) 1650010

4 (2016) 1650016

4 (2016) 1650018

1 (2016) 1650002

3 (2016) 1650015

3 (2016) 1650015

2 (2016) 1650008
Mahmoudvand, R. \&

Rodrigues, P. C.,

Missing value imputation in time series using Singular Spectrum Analysis

1 (2016) 1650005

Majumder, M., see

Ghosh, S.

4 (2016) 1650019

Massouh, F., see

Noura, B.

3 (2016) 1650013

Mbaka, C. K., Muiruri, P., Obiero, K. \&

Kisaka, M. O., An evaluation of grid-based rural electrification adoption dynamics in Meru-South sub-county

2 (2016) 1650010

Moghaddam, H., see Kunst, R.

4 (2016) 1650018

Muiruri, P., see Mbaka, C. K.

2 (2016) 1650010

Nor, N. M., see Khan, Z.

Noura, B., Dobrev, I., Khelladi, S. \& Massouh, F., 3D unsteady flow analysis around $a$ rotor blade of horizontal axis wind turbine-Rutland 503

3 (2016) 1650013

N'wuitcha, K., Igo, S. W.,

Atchonouglo, K., Palm, K.,

Banna, M. \& Zeghmati, B., Investigation on the combustion of volatile organic compounds for a cleaner planet

4 (2016) 1650016

Obiero, K., see Mbaka, C. K.

1 (2016) 1650003

Pal, M., see Ghosh, S.

Palm, K., see N'wuitcha, K.

Patidar, S., Jenkins, D. P. \& Simpson, S. A., Stochastic modelling techniques for generating
2 (2016) 1650010

4 (2016) 1650019

1 (2016) 1650003 
synthetic energy demand profiles

Pellegrini, G., see Bramati, M. C.

Pournasir, S., see Latifi, H.

Razali, R. B., see

Khan, Z.

Rodrigues, P. C., see Mahmoudvand, R.

Safari, A., see

Latifi, H.

Sayyareh, A., see

Fallahigilan, S.

Sharafi, A.,

Iranmanesh, H.,

Amalnick, M. S. \&

Abdollahzade, M.,

Financial

$\mathbf{3}(2016) 1650014$
$\mathbf{3}(2016) 1650012$
$\mathbf{1}(2016) 1650002$
$\mathbf{4}(2016) 1650016$
$\mathbf{1}(2016) 1650005$
$\mathbf{2}(2016) 1650009$

management of

Public Private

Partnership projects

using artificial

intelligence and

fuzzy model

Silva, E. S., see

Hassani, H.

2 (2016) 1650007

4 (2016) 1650020

Simpson, S. A., see

Patidar, S.

3 (2016) 1650014

Svensson, O., see

Li, W.

3 (2016) 1650015

Thomakos, D. D., see Alexopoulos, T. A.

2 (2016) 1650006

Waters, R., see Li, W.

Wirl, F., see Kunst, R.

Zeghmati, B., see

N'wuitcha, K.

3 (2016) 1650015

4 (2016) 1650018

1 (2016) 1650003 